Maße von subjektiven Auswahlentscheidungen abhängig ist es hingegen, wenn Konkordanzdemokratien und Mischsysteme mit stark plebiszitären Komponenten zu „vermessen“ sind. Die hier beobachteten Schwankungen sind gerade für die Schweizer Kantone beträchtlich; die Werte für die deutschen Bundesländer fallen demgegenüber - aufgrund der oben genannten Gründe - wesentlich homogener aus. Dass ein und derselbe Kanton innerhalb nur eines Jahres oder weniger Jahre von der Demokratie in den Bereich der Autokratie wechseln kann und umgekehrt, ist ein Befund, der erhebliche Zweifel an dem Messinstrument aufkommen lässt.

Die Übertragung des Vanhanen-Indexes auf die gliedstaatliche Ebene offenbart somit mehr als ein Vergleich von Nationalstaaten die dem Messinstrument innewohnenden Schwächen. Als Index, der beansprucht, durch die Heranziehung rein statistischer Daten ein hohes Maß an Objektivität aufzuweisen, unterbietet er diesen Anspruch doch so deutlich, dass man ihn nur mit allergrößter Vorsicht verwenden sollte. Ob es demgegenüber ein überzeugenderer Weg ist, qualitative Studien zu quantifizieren, sei hier dahingestellt. Da auch andere Indizes jeweils spezifische Schwächen aufweisen, kann zudem nicht ausgeschlossen werden, dass sich durch die kombinierte Anwendung von Indizes methodische Probleme nicht wechselseitig aufheben, sondern sogar kumulieren. Künftigen Forschungen zur Demokratiemessung kann man angesichts der hier berichteten Befunde jedenfalls nur raten, ihre Instrumente zunächst auf subnationaler Ebene - also gleichsam experimentell und in einer Art Laborsituation - sorgfältig zu testen, da eine solche Operation die Stärken und Schwächen verwendeter Instrumente besonders plastisch offenzulegen vermag.

\title{
Politisches Informationsverhalten in Österreich am Beispiel der Nationalratswahl 2006
}

\author{
Maria Beyrl, Peter Filzmaier und Flooh Perlot
}

Das Stichwort „Mediendemokratie“1 zeigt an, dass sich politische Prozesse an den Bedürfnissen der Massenmedien orientieren und Medien zentrale Kanäle der Politikvermittlung geworden sind. Was wir über Politik wissen beziehungsweise zu wissen glauben, wissen wir aus den Massenmedien.

Die These klingt plausibel, verallgemeinert aber. Die „Massenmedien“ sind in Wahrheit ein Konglomerat von unterschiedlichen Anbietern und technischen Übertragungsmethoden, das „Publikum“ ist ebenfalls eine soziodemographisch wie politisch gesehen heterogene Gruppe. Hinsichtlich des politischen Informationsverhaltens liegt es daher nahe, drei Differenzierungen zu versuchen: Haben unterschiedliche Medien unterschiedliche Bedeutung für das Publikum hinsichtlich des politischen Informationsverhaltens? Wer nutzt wel-

1 Vgl. etwa Heribert Schatz / Patrick Rössler / Jörg-Uwe Nieland, Politische Akteure in der Mediendemokratie: Einführung in die Thematik und Überblick über die Beiträge des Tagungsbandes, in: dies. (Hrsg.), Politische Akteure in der Mediendemokratie. Politiker in den Fesseln der Medien?, Wiesbaden 2002, S. $11-17$. 
che Angebote in welchem Ausmaß? Existieren Unterschiede zwischen „normalen“ Zeiten und Wahlkampfzeiten? Diese Fragen sollen anhand des politischen Informationsverhaltens in Österreich sowohl rund um die Nationalratswahl 2006 und die Wahl zum Europäischen Parlament (EP) 2004 als auch wahlunabhängig anhand von Daten aus dem Jahr 2007 sowie mit punktuellen internationalen Vergleichen im Folgenden behandelt werden.

\section{Mediale und politische Rahmenbedingungen der Politikvermittlung in Österreich vor und nach der Nationalratswahl 2006}

Unzweifelhaft hat sich die Politik- und Medienlandschaft Österreichs in den letzten Jahrzehnten sowohl strukturell als auch prozessual stark verändert. Zu den Brüchen zählten wechselnde Koalitionsregierungen (auf die Große Koalition folgte 2000 die erste Regierungszusammenarbeit von Österreichischer Volkspartei (ÖVP) und Freiheitlicher Partei Österreichs (FPÖ)), vorgezogene Neuwahlen, Aufstieg, Fall und Spaltung der Freiheitlichen Partei sowie eine Erweiterung des Parteienspektrums in Wahlkämpfen beziehungsweise auch in den Landtagen und im Bundesparlament. Parallel dazu kam es zu einer Schwächung und vermeintlichen Renaissance der Sozialpartnerschaft bis zur tiefen Gewerkschaftskrise 2006 im Zuge der BAWAG-Affäre ${ }^{2}$. Die im Herbst desselben Jahres stattfindende Nationalratswahl fand statt vor dem Hintergrund steigender Wechselwähleranteile, eines Mitgliederschwunds in Parteien und traditionellen Interessenorganisationen sowie zunehmender Verluste vor allem der Großparteien an die Gruppe der Nichtwähler ${ }^{3}$.

Im Medienbereich war es zu formal ähnlich dramatischen Umbrüchen gekommen. Nachdem 1995 die ersten beiden Privatradios in Österreich gestartet waren, folgte 1998 eine landesweite Einführung privater Radiosender. Die Politikvermittlung verlagerte sich in der Folge zunehmend von Meldungen in Nachrichtensendungen hin zu Kurzberichten in Musiksendungen und anderen Unterhaltungsformaten, und zwar auch in Programmen des ORF.

Im Fernsehen nahmen ebenso Privatsender ihre Arbeit auf, wobei es mit ATV nur eine terrestrische Alternative zum ORF gibt, die bundesweit sendet ${ }^{4}$. Mit ATV Aktuell und den ProSieben Austria Top News existieren neben der Zeit im Bild des ORF mittlerweile zwei private (werk-)tägliche Fernsehnachrichten, die stärker nach dem Prinzip des Infotainment gestaltet werden 5 . Für die Form der Politikvermittlung sind in diesem Zusammenhang

2 Die Bank für Arbeit und Wirtschaft (BAWAG), die sich im Besitz des Österreichischen Gewerkschaftsbundes (ÖGB) befand, geriet 2006 als Folge von spekulativen Geschäften in große finanzielle Bedrängnis, die auch den ÖGB schwer traf. Dieser musste als Konsequenz die Bank verkaufen.

3 Vgl. etwa Fritz Plasser / Peter A. Ulram / Gilg Seeber, Erdrutschwahlen: Momentum, Motive und neue Muster im Wahlverhalten, in: Fritz Plasser / Peter A. Ulram (Hrsg.), Wahlverhalten in Bewegung. Analysen zur Nationalratswahl 2002, Wien 2003, S. 97 - 157, oder Peter Filzmaier / Peter Hajek, Die Nationalratswahl 2006: Ergebnisse und Wahlverhalten, in: Peter Filzmaier / Peter Plaikner / Karl A. Duffek (Hrsg.), Mediendemokratie Österreich, Wien 2007, S. 63 - 90.

4 Falls nicht anders angegeben, beziehen sich alle Angaben auf den Stand Mitte 2007.

5 Vgl. Flooh Perlot, Alles wird neu und alles bleibt gleich - Politische Fernsehnachrichten in Österreich, in: Peter Filzmaier / Matthias Karmasin / Cornelia Klepp (Hrsg.), Politik und Medien - Medien und Politik, Wien 2006, S. 80 - 94. 
nicht allein unterschiedliche Ansprüche des privaten und öffentlich-rechtlichen Fernsehens zu beachten, sondern vor allem für Privatsender gilt auch eine radikale Ökonomisierung. Wesentlich ist zudem, dass die neuen elektronischen Medienangebote bisher kaum mehr als Nischenprodukte darstellen, da sie an Reichweite und Marktanteil mit niedrigen einstelligen Werten (noch) keine Konkurrenz für den ORF sind, der immer noch über mehr als 40 Prozent Marktanteil verfügt.

Auch im Printbereich kam es zu mehreren Neugründungen, die fast ausschließlich Boulevardcharakter aufwiesen und oft als Zweitblätter führender Medienhäuser und / oder als städtische Gratiszeitungen konzipiert waren ${ }^{6}$. Am 1. September 2006 - exakt einen Monat vor der Nationalratswahl - erschien zum ersten Mal die Tageszeitung „Österreich“. Diese präsentierte vor allem in der Politikberichterstattung einen plakativen Boulevard mit hohem Bildanteil und nur sehr vordergründigen Themenbezügen. Auffallend war zudem eine große Zahl veröffentlichter Meinungsumfragen.

Für die Politik- und Wahlkampfberichterstattung ist der Überhang der „Game-Zentrierung“ gegenüber der „Policy-Zentrierung“ (also eine Abkehr von sachpolitischen Inhalten hin zu einer sportiven Darstellung des Wahlkampfes) prägend ${ }^{7}$. Deutlich zum Ausdruck kam dies durch die mediale Konzentration auf Meta-Themen (die Auseinandersetzung von Parteien und Politikern an sich) im Vergleich zu Sachthemen: Der Wahlkampf wurde doppelt so oft medial behandelt wie das meistgenannte Sachthema ${ }^{8}$. Dieser Fokus auf MetaThemen ist kein österreichisches Phänomen, sondern findet sich international ${ }^{9}$.

Auf der politischen Akteursseite leisten Strategien der Inszenierung und kameragerecht aufbereitete „Pseudo-Ereignisse“ ihren Beitrag zur Game-Zentrierung in der politischen Berichterstattung. Zu beachten ist dabei, dass Inszenierung nichts Schlechtes sein muss; ohne Überschreitung der Aufmerksamkeitsschwelle der Medien und des Publikums ist jegliche Politikvermittlung vergebens ${ }^{10}$.

Auch das Wahlverhalten hat sich verändert: Die Zahl der spät entschlossenen Wähler so genannte late deciders treffen ihre Entscheidung erst zwei bis drei Wochen vor der Wahl, „last minute deciders“ in den letzten drei Tagen - ist auf 23 beziehungsweise 12 Prozent angestiegen. ${ }^{11}$ Damit gewinnt die Frage nach dem politischen Informationsverhalten im Wahlkampfzusammenhang immer mehr an Bedeutung.

6 Peter Plaikner, Die Medienlandschaft zwischen Donau-Wasserkopf und Alpen-Herrgottswinkel, in: Peter Filzmaier / Peter Plaikner / Karl A. Duffek (Hrsg.), Mediendemokratie Österreich, a.a.O. (Fn. 3), S. 179 - 210, S. $186 \mathrm{ff}$.

7 Günther, Pallaver / Clemens Pig / Günther Lengauer, Redaktionelle Politikvermittlung in österreichischen Wahlkämpfen, 1999 - 2006, in: Fritz Plasser / Peter A. Ulram (Hrsg.), Wechselwahlen. Analysen zur Nationalratswahl 2006, Wien 2006, S. 103 - 154, S. 126.

8 Ebenda, S. 133.

9 Günther Pallaver / Clemens Pig, Medienzentrierter Wahlkampf: Themen und Kandidaten in der Wahlkampfberichterstattung 2002, in: Fritz Plasser / Peter A. Ulram (Hrsg.), a.a.O. (Fn. 3), S. 55 -97, S. 85.

10 Vgl. Maria Beyrl/ Flooh Perlot, Politische Kommunikation in Österreich - Generalverdacht der Inszenierung? Ein Streifzug durch die österreichische Mediendemokratie, in: Österreichische Zeitschrift für Politikwissenschaft, 35. Jg. (2006), H. 4, S. $391-405$.

11 OGM - Österreichische Gesellschaft für Marketing/Donau-Universität Krems, Zeit im BildWahltagsbefragung zur Nationalratswahl 2006 im Auftrag des ORF, Wien 2006. 


\section{Die Mediendemokratie: Wichtigkeit und Glaubwürdigkeit politischer Informationen}

Informationen über Politik bezieht der weitaus größte Teil der Bevölkerung über Medien. Der Medienkonsum und insbesondere das Fernsehen sind die liebsten Freizeitbeschäftigungen in Österreich. Fast 99 Prozent der Haushalte verfügten 2006 über zumindest ein Fernsehgerät, doch lediglich in 80 bis 85 Prozent wird eine Tageszeitung gekauft beziehungsweise abonniert ${ }^{12}$. Österreicher sehen im Durchschnitt fast drei Stunden (163 Minuten) täglich fern (Stand 2006), darunter ORF-Programme 80 Minuten. Die zentralen politischen Nachrichtensendungen des ORF - Zeit im Bild 1 und Zeit im Bild 2 - verfügen normalerweise zusammen über eine Durchschnittsreichweite von etwa 1,6 bis über 2,0 Millionen Fernsehzuschauer. Das entspricht bis zu 30 Prozent der Wahlberechtigten. Von diesen lesen (Stand 2003) 30 Prozent täglich den Politikteil einer Zeitung, weitere 25 Prozent mehrmals die Woche. Rund 45 Prozent tun das seltener oder - zu rund einem Viertel - fast nie, wobei entsprechende Werte für Erwachsene seit 1960 weitgehend konstant sind. Die Reichweiten der Nachrichtenprogramme in den Privatsendern betragen jeweils nur etwa zwei bis drei Prozent und sind lediglich bei den unter 30-Jährigen signifikant höher; an einem beispielhaft ausgewählten Tag im Mai 2007 erreichte etwa ATV Aktuell rund 53.000 Seher, während über 970.000 die Zeit im Bild (die zeitlich direkt an die ATVNachrichten anschließt) einschalteten ${ }^{13}$.

Generell, das heißt unabhängig von der Wahlkampfzeit und politischen Ausnahmeereignissen, beziehen die meisten Österreicher ihre politischen Informationen überwiegend aus dem Fernsehen, das auch als am glaubwürdigsten gilt. Etwa drei Viertel (71 Prozent; Stand Januar 2007) bezeichnen das Fernsehen als wichtigste politische Informationsquelle ${ }^{14}$ (vgl. Tabelle 1). Die Zahl der Mediennutzer, für die Politik via Bildschirm an erster Stelle steht, ist damit seit den neunziger Jahren relativ konstant ${ }^{15}$. Ebenso konstant folgen Zeitungen trotz der Möglichkeit von Mehrfachnennungen mit nur 54 Prozent Nutzung als primäre Politikquelle zu Jahresbeginn 2007 mit deutlichem Abstand auf dem zweiten Platz. Radikal an Bedeutung verliert der Hörfunk: 2003 war er noch für 38 Prozent, 2007 nur noch für 21 Prozent die zentrale Quelle.

Radio- und Printjournalisten könnten einwenden, dass die Wichtigkeit politischer Informationsquellen von der inhaltlichen Qualität unabhängig ist, weil sie sehr von der durch individuelle Tagesabläufe bedingten Nutzungshäufigkeit abhängt beziehungsweise in einer Fernsehgesellschaft lediglich allgemeine Entwicklungen widerspiegelt. Für Medienwirkungen entscheidender wäre demzufolge die Glaubwürdigkeit. Diesbezüglich liegt jedoch das Fernsehen klar vor den Zeitungen und dem abgeschlagenen Hörfunk: Rund 40 bis 50 Pro-

12 Vgl. ORF-Medienforschung 2006 und Media-Analyse 2006.

13 Vgl. ORF-Medienforschung unter http://mediaresearch.orf.at beziehungsweise die auf http:// www.atv.at veröffentlichten Daten, Abruf für beide am 28. Mai 2007.

14 OGM - Österreichische Gesellschaft für Marketing / DUK - Donau-Universität Krems, Politisches Informationsverhalten in Österreich, unveröffentlichte Repräsentativumfrage, Wien / Krems 2007.

15 Fritz Plasser / Peter A. Ulram, Öffentliche Aufmerksamkeit in der Mediendemokratie, in: Fritz Plasser (Hrsg.), Politische Kommunikation in Österreich. Ein praxisnahes Handbuch, Wien 2004, S. 37 - 100, S. 74 ff., sowie Peter Filzmaier, Wahlen und politischer Wettbewerb in der Mediengesellschaft, in: Forum Politische Bildung (Hrsg.), Von Wahl zu Wahl, Wien / München 2004, S. 13 - 21, S. 14. 
zent - 51 Prozent 2003 sowie bei einer deutlich höheren Zahl von Antwortverweigerungen 39 Prozent 2007 - halten das Fernsehen, das trotz einer zu vermutenden Verschlechterung in den letzten Jahren mit knapp 15 beziehungsweise 24 Prozent deutlich vor den Zeitungen rangiert, für am glaubwürdigsten. Trotz der einmaligen Sonderstellung der Kronen Zeitung und ihrer Wirkung in Wahlkämpfen ${ }^{16}$ müssen in diesem Zusammenhang die politischen Einflussmöglichkeiten von Printmedien deutlich relativiert werden. Allerdings haben Zeitungen in der jüngsten Vergangenheit an politischer Glaubwürdigkeit (zurück-)gewonnen, während das Fernsehen - alle Daten beziehen sich zwangsläufig vor allem auf den öffentlich-rechtlichen ORF - zunehmend um diese kämpfen muss. Immerhin sind die Zeitungen trotz einer geringeren Auskunftsbereitschaft der Befragten zwischen 2003 und 2007 deutlich glaubwürdiger geworden. Offensichtlich ist auch, dass die Politikberichterstattung im Radio in ihrer Wichtigkeit einen radikalen Bedeutungsverlust erfahren hat, während sie hinsichtlich der Glaubwürdigkeit auf niedrigem Niveau stagniert. Zu beachten ist für alle Daten, dass keine Unterscheidung von Informations- und Unterhaltungssendungen im Fernsehen - Stichwort talk show politics -, von Qualitäts- und Boulevardzeitungen sowie

\begin{tabular}{|c|c|c|c|c|c|c|c|c|c|c|}
\hline \multicolumn{11}{|c|}{$\begin{array}{l}\text { Tabelle 1: Bedeutung (1961 bis 2007) und subjektive Glaubwürdigkeit (1989 bis 2007) medialer } \\
\text { politischer Informationsquellen in Österreich (Angaben in Prozent) }\end{array}$} \\
\hline & \multicolumn{5}{|c|}{$\begin{array}{l}\text { Befragte bezeichnen als primäre politische } \\
\text { Informationsquelle }\end{array}$} & \multicolumn{5}{|c|}{ Befragte halten für besonders glaubwürdig } \\
\hline & 1961 & 1995 & 2001 & 2003 & 2007 & 1989 & 1995 & 1999 & 2003 & 2007 \\
\hline Fernsehen & 11 & $\begin{array}{r}69 \\
(+58)\end{array}$ & $\begin{array}{r}79 \\
(+10)\end{array}$ & $\begin{array}{r}75 \\
(-4)\end{array}$ & $\begin{array}{r}71 \\
(-4)\end{array}$ & 56 & $\begin{array}{r}47 \\
(-9)\end{array}$ & $\begin{array}{r}54 \\
(+7)\end{array}$ & $\begin{array}{r}51 \\
(-3)\end{array}$ & $\begin{array}{r}39 \\
(-12)\end{array}$ \\
\hline Tageszeitung & 61 & $\begin{array}{r}44 \\
(-17)\end{array}$ & $\begin{array}{r}52 \\
(+8)\end{array}$ & $\begin{array}{r}49 \\
(-3)\end{array}$ & $\begin{array}{r}54 \\
(+5)\end{array}$ & 16 & $\begin{array}{r}20 \\
(+4)\end{array}$ & $\begin{array}{r}14 \\
(-6) \\
\end{array}$ & 14 & \begin{tabular}{|r}
24 \\
$(+10)$ \\
\end{tabular} \\
\hline Hörfunk & 59 & - & 45 & $\begin{array}{r}38 \\
(-7) \\
\end{array}$ & $\begin{array}{r}21 \\
(-18) \\
\end{array}$ & 9 & $\begin{array}{r}8 \\
(-1) \\
\end{array}$ & $\begin{array}{r}7 \\
(-1) \\
\end{array}$ & $\begin{array}{r}8 \\
(+1) \\
\end{array}$ & $\begin{array}{r}5 \\
(-3) \\
\end{array}$ \\
\hline Zeitschrift & - & - & - & - & 3 & 5 & $\begin{array}{r}7 \\
(+2) \\
\end{array}$ & $\begin{array}{r}3 \\
(-4) \\
\end{array}$ & $\begin{array}{r}2 \\
(-1) \\
\end{array}$ & $\begin{array}{r}0 \\
(-2) \\
\end{array}$ \\
\hline Gespräche & 7 & $\begin{array}{r}12 \\
(+5) \\
\end{array}$ & $\begin{array}{r}11 \\
(-1) \\
\end{array}$ & $\begin{array}{r}8 \\
(-3) \\
\end{array}$ & $\begin{array}{r}5 \\
(-3) \\
\end{array}$ & 5 & $\begin{array}{r}16 \\
(+6) \\
\end{array}$ & $\begin{array}{r}12 \\
(-4) \\
\end{array}$ & - & $\begin{array}{r}2 \\
(-10) \\
\end{array}$ \\
\hline Internet & - & - & - & 4 & $\begin{array}{r}9 \\
(+5) \\
\end{array}$ & - & - & - & 2 & $\begin{array}{r}4 \\
(+2) \\
\end{array}$ \\
\hline \multicolumn{11}{|c|}{$\begin{array}{l}\text { Gefragt war jeweils nach wichtigen Informationsquellen über österreichische Politik - mit der Möglich- } \\
\text { keit von Mehrfachnennungen - und nach der glaubwürdigsten Quelle (ohne Mehrfachnennung). Zu } \\
\text { berücksichtigen ist, dass es sowohl aufgrund leicht abweichender Frageformulierungen im Zeitverlauf als } \\
\text { auch infolge der differierenden Zahl von unentschiedenen Antworten, Antwortverweigerungen oder feh- } \\
\text { lenden Angaben zu Verzerrungen kommen kann. } \\
\text { Quellen: Für bis } 2004 \text { Fessel-GfK, bundesweite (telefonische) Repräsentativumfragen, n = } 1.000 \text { bis } \\
\text { 1.500, zitiert in: Fritz Plasser / Peter A. Ulram, Das österreichische Politikverständnis. Von der Konsens- } \\
\text { zur Konfliktkultur, Wien 2002, S. } 39 \text { ff., und dies., a.a.O. (Fn. 15), S. 74, S. 92, für } 2007 \text { Österreichi- } \\
\text { sche Gesellschaft für Marketing (OGM) im Auftrag der Donau-Universität Krems, bundesweite (telefo- } \\
\text { nische) Repräsentativumfrage Ende Januar, n }=1.000 \text {. }\end{array}$} \\
\hline
\end{tabular}

16 Peter Filzmaier, Campaigning and Media in Austria: Lessons to be Learned from the „HPM Phenomenon" in the European Parliamentary Elections, in: Michaela Maier / Jens Tenscher (Hrsg.), Campaigning in Europe - Campaigning for Europe. Political Parties, Campaigns, Mass media and the European Parliament Elections 2004, London 2006, S. 323 - 338, S. 330 ff. 
von Nachrichten- und Flächenprogrammen im Radio stattfindet. Es handelt sich demnach um Durchschnittswerte, die in allen Medien durch eine zunehmende „Boulevardisierung“ der Politikvermittlung geprägt sind.

Auch international gesehen ist die Bedeutung des Fernsehens hoch: Fernsehnachrichten sind für etwa drei Viertel der Bevölkerung in Nordamerika und Westeuropa die primäre Informationsquelle über Politik sowie das dominante Medium der Wahlkommunikation ${ }^{17}$. Es liegt auch hinsichtlich seiner täglichen Nutzung in allen EU-Staaten vor den Tageszeitungen $^{18}$. Im Gegensatz zu dieser nahezu universellen Verbreitung schwanken die Auflagenzahlen und Reichweiten von Tageszeitungen stark. Hohe Auflagenzahlen finden sich etwa in Skandinavien, in mitteleuropäischen Ländern wie Deutschland, Schweiz und Österreich, in Großbritannien und Japan. In den USA sind die Auflagenzahlen hingegen vergleichsweise gering ${ }^{19}$.

Nach soziodemographischen Indikatoren ergibt sich folgendes Bild (vgl. Tabelle 2): Nach dem Geschlecht bestehen weder hinsichtlich der Wichtigkeit noch für die Glaubwürdigkeit politischer Informationsquellen Unterschiede. Altersmäßig steigt die Wichtigkeit des Fernsehens mit den Lebensjahren von zwei Drittel für unter 30-Jährige auf über drei Viertel (76 Prozent) für über 50-Jährige. Analog dazu sind Zeitungen für die 50-plus-Generation eindeutig am wichtigsten. Das Internet hingegen nennt knapp ein Fünftel der bis zu 30 Jahre alten Österreicher als wichtige Politikquelle, während das nur für eine von 100 aus der Gruppe der über 50 Jahre alten zutrifft. Die höchste abgeschlossene Schulbildung beeinflusst die Wichtigkeit insofern, als formal niedriger gebildete Pflichtschulabsolventen für politische Informationen deutlich mehr das Fernsehen (mit 81 Prozent Wichtigkeit) und das Radio nutzen. Zeitungen und Internet sind demgegenüber für besser Gebildete wichtiger.

Die Glaubwürdigkeit des Fernsehens ist für Absolventen von Allgemeinbildenden Höheren Schulen (AHS, entsprechen ungefähr deutschen Abiturienten) mit 30 Prozent im Vergleich zu über 40 Prozent für alle anderen Schultypen am geringsten. Umgekehrt verhalten sich die Zahlen bei Zeitungen, das heißt, diese sind unter den AHS-Absolventen besonders glaubwürdig beziehungsweise mit 29 Prozent mit dem Fernsehen nahezu gleichrangig. Nach dem Alter ist erwartungsgemäß das Internet in der jüngsten Bevölkerungsgruppe und unter den AHS-Absolventen aus der Gruppe der Nutzer nicht nur am wichtigsten, sondern auch am glaubwürdigsten.

Bezogen auf die bekundete Parteipräferenz ergeben sich die signifikantesten Differenzen zwischen Anhängern der Grünen und der FPÖ: Grüne räumen dem Fernsehen einen verhältnismäßig geringen und den Zeitungen einen großen Stellenwert ein. Vier von fünf FPÖ-Anhängern betonen hingegen die Wichtigkeit des Fernsehens, nur einer von zweien jene der Zeitungen. Auffallend ist der lineare Bedeutungsverlust des Radios in der Reihenfolge SPÖ-, ÖVP-, Grün- und FPÖ-Wähler von 27 auf 13 Prozent Wichtigkeit. Ebenso nach Parteien sinkt die Glaubwürdigkeit des Fernsehens beziehungsweise steigt jene der

17 Fritz Plasser / Gunda Plasser, Globalisierung der Wahlkämpfe. Praktiken der Campaign Professionals im weltweiten Vergleich, Wien 2003, S. 255, S. $242 \mathrm{f}$.

18 Josef Seethaler / Gabriele Melischek, Pressekonzentration in Österreich im europäischen Vergleich, in: Österreichische Zeitschrift für Politikwissenschaft, 35. Jg. (2006), H. 4, S. $337-360$, S. 348 .

19 Fritz Plasser / Gunda Plasser, a.a.O. (Fn. 17), S. 239. 


\begin{tabular}{|c|c|c|c|c|c|c|c|c|c|c|}
\hline & & & & & $\infty$ & & 0 & 6 & \multirow{25}{*}{ 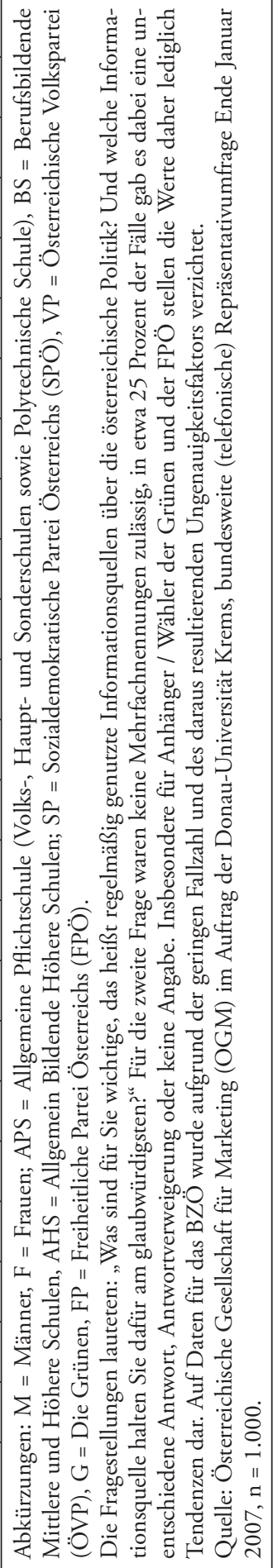 } \\
\hline & & $\overrightarrow{\breve{v}}$ & 0 & ले & ¿ & $n$ & $\sim$ & - & $\infty$ & \\
\hline & & & & $F$ & 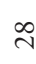 & - & & 0 & $a$ & \\
\hline & & & $\vec{n}$ & n & 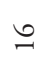 & $\wedge$ & 0 & - & $\sim$ & \\
\hline & & $\stackrel{\infty}{\Xi}$ & & m & ন & $n$ & v & - & $a$ & \\
\hline & & $\begin{array}{l}\bar{t} \\
\overrightarrow{0}\end{array}$ & $\infty$ & $\stackrel{\sim}{F}$ & $\widetilde{\sim}$ & t & D & $n$ & $\psi$ & \\
\hline & & 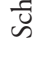 & 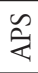 & 위 & సి & $\infty$ & 0 & 0 & - & \\
\hline & & & in & ले & तี & $\checkmark$ & 0 & $N$ & - & \\
\hline & & 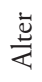 & îे & 와 & $\vec{\sim}$ & ๑ & - & - & ○ & \\
\hline & & & $\infty$ & $\stackrel{\infty}{n}$ & $\stackrel{\infty}{\sim}$ & $n$ & & - & $\infty$ & \\
\hline & & 荀 & I & 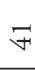 & $\vec{\sim}$ & in & - & $\sim$ & $\psi$ & \\
\hline & & $\begin{array}{l}\vec{u} \\
\ddot{U} \\
\ddot{u}\end{array}$ & $\Sigma$ & $\hat{n}$ & $\approx$ & in & 0 & $N$ & $n$ & \\
\hline U & & & $\bar{I}$ & N & fr & $\stackrel{m}{=}$ & & $\infty$ & 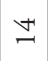 & \\
\hline$s$ & $\frac{\vec{v}}{\vec{v}}$ & 莺 & 0 & $\stackrel{m}{n}$ & $\sqrt{n}$ & $\stackrel{?}{\simeq}$ & $E$ & $n$ & $\hat{\imath}$ & \\
\hline & 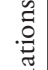 & 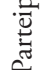 & 5 & $\infty$ & ถิ & $\tilde{N}$ & & $n$ & $\simeq$ & \\
\hline$\Xi$ & 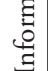 & & $\vec{n}$ & e & F & તิ & + & 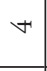 & $\wedge$ & \\
\hline 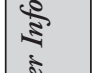 & 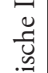 & $\stackrel{\infty}{\Xi}$ & & $\widehat{6}$ & రु & $\vec{\sim}$ & & $\checkmark$ & $\vec{\sim}$ & \\
\hline 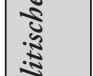 & $\begin{array}{l}:= \\
0 \\
\vdots \\
0\end{array}$ & 矛 & $\tilde{q}$ & R & 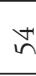 & $\stackrel{\infty}{-}$ & tr & $\bullet$ & $\lambda$ & \\
\hline 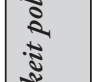 & : & 氙 & $\tilde{z}$ & $\infty$ & 冓 & নे & & $m$ & $m$ & \\
\hline 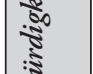 & $\begin{array}{l}\bar{y} \\
\tilde{u} \\
\tilde{v}\end{array}$ & & $\bar{n}$ & م & ฉ & $\widetilde{\approx}$ & & 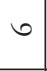 & - & \\
\hline$\frac{\sqrt{3}}{3}$ & 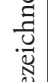 & 苞 & in & $\infty$ & $\bar{n}$ & $\vec{\sim}$ & 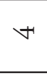 & $n$ & $\stackrel{n}{\sim}$ & \\
\hline $\begin{array}{ll}5 \\
5 \\
5 \\
5\end{array}$ & $\begin{array}{l}0 \\
0 \\
\tilde{5} \\
0\end{array}$ & & ते & 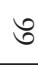 & 冓 & $\stackrel{\bullet}{-}$ & & 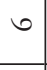 & $\beth$ & \\
\hline $\begin{array}{l}50.5 \\
3 \\
3 \\
3 \\
3\end{array}$ & $\begin{array}{l}\stackrel{\mathscr{J}}{\sqcup} \\
\check{D}\end{array}$ & 苞 & I & ה & in & సิ & & 6 & $\lambda$ & \\
\hline$\Sigma$ & & & $\Sigma$ & $\Gamma$ & in & ปี & . & $m$ & $\exists$ & \\
\hline 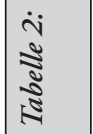 & & & & & & 管 & 氕 & 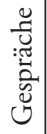 & 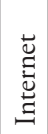 & \\
\hline
\end{tabular}


Tageszeitungen. Jeder zweite Anhänger der Sozialdemokratie und nur etwa jeder siebte der FPÖ (16 Prozent) vertraut dem Fernsehen für politische Informationen am meisten. Umgekehrt ordnen 30 Prozent der Grünen- und 40 Prozent der FPÖ-Wähler Zeitungen die meiste Glaubwürdigkeit zu. Das Phänomen erklärt sich aus der Tatsache, dass unter den Grünen besonders viele formal hochgebildete Leser von Qualitätszeitungen sind und die FPÖ-Wähler traditionell einen starken Anteil von Lesern der Kronen Zeitung aufweist.

\section{Informationsverhalten im Nationalratswahlkampf 2006}

Die ORF-Fernsehdiskussionen im Nationalratswahlkampf 2006 - es saßen sich jeweils zu zweit die Parteichefs oder deren Vertreter von allen im Parlament vertretenen Gruppen gegenüber - wurden insgesamt von 3,626 Millionen Österreichern (59,5 Prozent der Bevölkerung beziehungsweise der Wahlberechtigten ab 18 Jahren) wenigstens zum Teil gesehen. Bis zu 1.318.000 Zuschauer oder 21,6 Prozent der Wahlberechtigten verfolgten die Fernsehkonfrontation der Kanzlerkandidaten von ÖVP und SPÖ mit Wolfgang Schüssel und Alfred Gusenbauer am 21. September 2006 in ORF 2. Bis zu 1.442.000 Zuschauer (23,6 Prozent) gab es für die Diskussion aller Spitzenkandidaten der fünf Parlamentsparteien am 28. September 2006 - also drei Tage vor der Wahl - in ORF 2. Im Durchschnitt waren 1,264 Millionen (20,7 Prozent) an der „Elefantenrunde“ interessiert. Das entsprach einem Marktanteil von 47 Prozent. Die Zeit im Bild des ORF am Wahltag um 19.30 Uhr verfolgten auf ORF 1 und ORF 2 insgesamt 2,18 Millionen, das heißt mehr als 46 Prozent oder fast jeder zweite Wähler. Der Marktanteil betrug damit 76 Prozent. Die Zeit im Bild am Wahlsonntag des 1. Oktober 2006 war somit die reichweitenstärkste Ausgabe seit der vergangenen Nationalratswahl 2002. Insgesamt sahen vier Millionen Menschen wenigstens kurz die Wahlberichterstattung im ORF. Im Durchschnitt erreichte ORF 2 während der sechs Stunden Sendezeit zur Wahl 2006 knapp 1,3 Millionen Zuschauer und einen Marktanteil von 55 Prozent, während sich nur 89.000 via ATV bei der dortigen Sondersendung zur Nationalratswahl über die Ergebnisse informierten.

Über die Wichtigkeit und Glaubwürdigkeit politischer Informationsquellen liegen auf Bundesebene Daten für die Wahl zum Europäischen Parlament (EP) 2004 vor, die mit jenen für den Nationalratswahlkampf 2006 von der Fragestellung her vergleichbar sind. Zu beachten ist allerdings die extrem unterschiedliche Wahlbeteiligung von 42,4 beziehungsweise 78,5 Prozent. Diese erklärt auch bemerkenswerte Unterschiede: Während hinsichtlich der Wichtigkeit der einzelnen Medien, um sich über Politik und Wahlkampf zu informieren, zwischen den allgemeinen Werten zu Jahresbeginn 2007 und der (Intensiv-)Wahlkampfzeit im September 2006 fast keine Abweichungen bestehen (71 Prozent bezeichnen das Fernsehen als Primärquelle, 54 beziehungsweise 55 Prozent Zeitungen und 22 beziehungsweise 21 Prozent das Radio), war für EP-Wähler das Fernsehen klar weniger bedeutend: Nur 56 Prozent nannten es als hauptsächliche Informationsquelle für den Wahlkampf um Sitze im EP. Gleichermaßen auffallend war der höhere Stellenwert von Gesprächen mit zwölf Prozent gegenüber ansonsten niedrigen einstelligen Prozentwerten ${ }^{20}$.

20 OGM - Österreichische Gesellschaft für Marketing, Zeit im Bild-Wahltagsbefragung zu den österreichischen Wahlen zum Europäischen Parlament im Auftrag des ORF, Wien 2004, sowie Peter Filzmaier, a.a.O. (Fn. 16), S. 330. 
Hinsichtlich der Glaubwürdigkeit waren die Unterschiede geringer ausgeprägt. Jeweils rund ein Drittel hielt sowohl in Wahlkampfzeiten als auch allgemein das Fernsehen für am glaubwürdigsten. Tageszeitungen verfügten in Wahlkämpfen generell und vor allem im Nationalratswahlkampf 2006 über vergleichsweise geringe Vertrauenswerte. Untypisch hoch war mit zehn Prozent der Anteil von Gesprächen als glaubwürdigste Quelle im EP-Wahlkampf 2004. Es drängt sich die Hypothese auf, dass die angesichts der reduzierten Wahlbeteiligung offenbar überdurchschnittlich interessierten Wähler der interpersonalen Kommunikation über Politik mehr Bedeutung zumessen.

Konkret auf die Zeit des EP- und Nationalratswahlkampfs 2004 und 2006 bezogen (vgl. Tabelle 3) zeigten sich als Phänomene für das politische Informationsverhalten sowohl Übereinstimmungen als auch Abweichungen von den langjährigen Werten aus der wahlkampffreien Zeit ${ }^{21}$ : Nach der Wichtigkeit liegt das Fernsehen auch in Wahlkampfzeiten weit vor den Tageszeitungen. Fast drei Viertel (71 Prozent) der Wähler beziehungsweise Wahlberechtigten nannten es im Nationalratswahlkampf als wichtige Informationsquelle, während nur 55 Prozent dasselbe von Tageszeitungen behaupteten. Summiert man die Werte für Tages- mit jenen für Wochen- beziehungsweise Monatszeitungen, ergibt sich trotzdem ein Bedeutungsvorsprung des elektronischen Mediums Fernsehen gegenüber den Printmedien von elf Prozentpunkten.

\begin{tabular}{|l|l|l|c|c|c|}
\hline \multicolumn{7}{|c|}{ Tabelle 3: Wichtigkeit und Glaubwürdigkeit von Massenmedien im EP-und Nationalratswahl- } \\
kampf in Österreich 2004 und 2006 sowie 2007 (Angaben in Prozent)
\end{tabular}

Am wichtigsten war das Fernsehen - mit allerdings nicht extremen Abweichungen - für Wähler der traditionellen Großparteien ÖVP und SPÖ, für Frauen, für ältere Menschen und Bewohner ländlicher Gebiete (vgl. Tabelle 4). Auch diesbezüglich ergab sich eine Übereinstimmung mit den Daten für die Nicht-Wahlkampfzeit. Tageszeitungen hatten demge-

21 OGM - Österreichische Gesellschaft für Marketing, Zeit im Bild-Wahltagsbefragungen zur Nationalratswahl im Auftrag des ORF, Wien 2006, sowie Peter Filzmaier / Peter Hajek, a.a.O. (Fn. 3), S. $81 \mathrm{ff}$. 
genüber eine überdurchschnittliche Bedeutung für über 50-Jährige sowie für Arbeiter und Angestellte beziehungsweise für Grünen-Wähler. 22 Prozent bezeichneten das Radio als wichtige Quelle für Wahlkampfinformationen, zehn Prozent - wiederum mit sehr vielen Grünen (19 Prozent) - das Internet. Letzteres hatte zugleich unter jüngeren und städtischen Wählern die höchsten Glaubwürdigkeitswerte.

Während es kaum geschlechtsspezifische Unterschiede gibt, nimmt - auf die Politikberichterstattung zur Nationalratswahl 2006 bezogen - überraschenderweise die Glaubwürdigkeit des Fernsehens mit steigendem Lebensalter ab, so von 38 Prozent als glaubwürdigste Informationsquelle für die unter 30-Jährigen auf 28 Prozent für die über 50-Jährigen, ohne dass es vergleichbare Erfahrungen außerhalb von Wahlkämpfen gibt. Bezogen auf Berufsgruppen kann vereinfacht festgestellt werden, dass analog zum Vertrauensverlust unter den älteren Wählern primär Pensionäre dem Fernsehen misstrauen. Regional vertrauen Landbewohner (36 Prozent) dem Fernsehen mehr als Städter (28 Prozent). Nach der Parteipräferenz ist das Fernsehen bei ÖVP- und FPÖ-Anhängern für jeweils rund 40 Prozent am glaubwürdigsten, doch rangiert die den ORF im Vorwahlkampf scharf kritisierende SPÖ nur knapp dahinter. Deutlich geringer ist die Glaubwürdigkeit des Fernsehens lediglich bei Wählern der Grünen mit 29 Prozent. In teilweisem Gegensatz dazu ergaben sich für Tageszeitungen kaum Differenzen nach dem Alter oder der Region. Auffallend ist lediglich die signifikant hohe Glaubwürdigkeit der Tageszeitungen unter den Grünen-Anhängern, die in dieser Gruppe mit 28 Prozent an jene des Fernsehens heranreicht und bei der Wählerstruktur mit einem hohen formalen Bildungsgrad zusammenhängt.

Das Internet war im Nationalratswahlkampf wiederum für Männer, junge Wähler und Städter führend. Besonders beeindruckend ist, dass sowohl hinsichtlich der Wichtigkeit von politischen Informationsquellen als auch bezogen auf ihre Glaubwürdigkeit persönliche Gespräche anders als in den Wahlen zum EP fast gar keinen Stellenwert hatten. Die Zahl der entsprechenden Nennungen war mit höchstens zwei Prozent und häufigen Leermeldungen statistisch nicht erfassbar. Selten wurde der Begriff Mediendemokratie anschaulicher dokumentiert. Nicht nachgewiesen sind dadurch jedoch Einflüsse auf das Wahlverhalten. Oft zeigt sich, dass die Bedeutung medialer Kampagnen der Parteien und / oder Kandidaten auf das Wahlergebnis relativ gering ist. Mit anderen Worten: Hätten die Kampagnen nicht stattgefunden, wäre die Stimmenverteilung unter Umständen nicht wesentlich anders gewesen. Diese verblüffende Feststellung ${ }^{22}$ erklärt sich, weil einerseits schwierig zu steuernde Prozesse der persönlichen Interaktion in einem komplexen sozialen Umfeld das Wahlverhalten prägen. Andererseits gibt es laufend nicht vorhersehbare Ereignisse, die als intervenierende Variablen auftreten.

\section{Internationaler Vergleich: Deutschland und die USA}

Ein exakter Vergleich von Daten aus anderen Ländern mit den vorliegenden Ergebnissen aus Österreich ist nicht möglich, da aus nahe liegenden Gründen auf Sekundärquellen zugegriffen werden muss, die auf unterschiedlichen Fragestellungen, Methoden etc. beruhen.

22 Vgl. David Farell / Rüdiger Schmitt-Beck, Do Political Campaigns Matter? Campaign Effects in Elections and Referendums, London 2002. 
Dennoch lassen sich an exemplarischen Fällen aus Deutschland und den USA zumindest ähnliche Tendenzen zeigen, die für eine relativ große internationale Konvergenz in der politischen Mediennutzung sprechen ${ }^{23}$. Vor allem die Dominanz des Fernsehens lässt sich international eindeutig belegen ${ }^{24}$.

So nannten 200684 Prozent von in Deutschland Befragten das Fernsehen als die häufigste Quelle für tagesaktuelle Informationen, Zeitungen landeten mit 62 Prozent auf Platz zwei, deutlich vor dem Radio und dem Internet ${ }^{25}$. In Wahlkampfzeiten zeigt sich ein vergleichbares Bild, das in der Ausprägung noch deutlicher ist. Im Bundestagswahlkampf 2002 holten sich 57 Prozent der Deutschen ihre Informationen hauptsächlich aus dem Fernsehen, andere Massenmedien waren weit abgeschlagen (Tageszeitungen: 25, Radio / Zeitschriften: 5, Internet: 3 Prozent) ${ }^{26}$.

In den USA beziehen über 70 Prozent der Bevölkerung politische Informationen in Wahlkampfzeiten aus dem Fernsehen ${ }^{27}$. Zwar sind die Werte rückläufig, liegen aber nach wie vor auf einem sehr hohen Niveau im Vergleich zu anderen Medien (vgl. Tabelle 5).

\begin{tabular}{|c|c|c|c|c|c|}
\hline \multicolumn{6}{|c|}{$\begin{array}{l}\text { Tabelle 5: Primäre Informationsquellen über den US-Wahlkampf, } 1960 \text { bis } 2004 \\
\text { (Angaben in Prozent) }\end{array}$} \\
\hline & Fernsehen & Tageszeitungen & Radio & Magazine & Internet \\
\hline 1960 & 51 & 57 & 34 & 8 & \\
\hline 1992 & 82 & 57 & 12 & 9 & \\
\hline 1996 & 72 & 60 & 19 & 11 & 3 \\
\hline 2000 & 70 & 39 & 15 & 4 & 11 \\
\hline 2004 & 76 & 46 & 22 & 6 & 21 \\
\hline \multicolumn{6}{|c|}{$\begin{array}{l}\text { Gefragt war nach den Informationsquellen über den Vorwahlkampf. Mehrfachnennung erlaubt. Gleiche } \\
\text { Trends zeigen Erhebungen zu den Informationsquellen über Wahlkämpfe in den USA - siehe dazu The } \\
\text { Pew Research. Center for The People and The Press, Social networking and online videos take off. Inter- } \\
\text { net's broader role in campaign 2008, Washington D.C. } 2008 \text {. } \\
\text { Quellen: National Television Association and the National Association of Broadcasters beziehungsweise } \\
\text { The Pew Research Center for The People and The Press, Media Consumption and Believability Study } \\
\text { 2004, Washington D.C. 2004, zitiert nach Peter Filzmaier / Fritz Plasser, a.a.O. (Fn. 27), S. 105. }\end{array}$} \\
\hline
\end{tabular}

Es ist absehbar, dass das Internet weiter an Bedeutung gewinnen wird. Die Zahl seiner Nennungen im Informationszusammenhang hat sich in den USA seit Mitte der 1990er Jahre auf 21 Prozent erhöht. Das Internet wird - auch durch die Verbreitung von Breitbandzugängen und unlimitierten Datenmengen - mehr und mehr zu einem alltäglichen Massenmedium $^{28}$, wobei es vor allem die Quelle der jungen Generation ist. Besonders Jugendliche und Menschen unter 30 Jahren gehören auch international gesehen zu seinen

23 Vgl. oben beziehungsweise Daten bei Josef Seethaler / Gabriele Melischek, a.a.O. (Fn. 18), und Fritz Plasser / Gunda Plasser, a.a.O. (Fn. 17).

24 Fritz Plasser / Peter A. Ulram, a.a.O. (Fn. 15), S. 75.

25 Allensbacher Computer- und Technikanalyse (ACTA) 2006.

26 Vgl. http://www.presseportal.de/pm/7840/382294/zdf/, Abruf am 26. März 2008.

27 Peter Filzmaier / Fritz Plasser, Politik auf amerikanisch. Wahlen und politischer Wettbewerb in den USA, Wien 2005, S. 105.

28 Andrew Chadwick, Internet politics. States, Citizens, and New Communication Technologies, New York 2006, S. 8. 
Nutzern, sowohl allgemein als auch im speziellen politischen Kontext. Demgegenüber steht eine zunehmende Überalterung des Publikums bei Fernsehnachrichten. In den USA leiden daran vor allem die traditionellen Abendnachrichten der großen Fernsehsender ABC, CBS und NBC (sowie FoxTV); die Hälfte ihres Stammpublikums ist über 61 Jahre alt $^{29}$. Das entspricht in etwa dem Altersschnitt der Zeit in Bild 1-Seher in Österreich, während allgemein etwa die Hälfte des ORF-Publikums über 50 Jahre zählt ${ }^{30}$.

Die Bedeutung des Internets bei jungen Menschen muss jedoch in Relation zu der Bedeutung der anderen Medien gesehen werden und darf vor allem nicht darüber hinwegtäuschen, dass die politische Informationsnutzung im Netz insgesamt vergleichsweise gering ist. Knapp 50 Prozent der 14- bis 19-Jährigen in Deutschland geben an, das Internet nie für politische Informationen zu nutzen, bei den 20- bis 29-Jährigen sind es sogar über 60 Prozent ${ }^{31}$; eine andere Studie spricht gar von 83 Prozent der Nutzer, die „nur ,etwas, kaum oder gar nicht' an politischen Informationen interessiert (...) “32 sind.

Die Glaubwürdigkeit politischer Informationsquellen wird auch international vom Fernsehen dominiert, das aber zunehmend an Boden verliert - jedoch nicht zugunsten eines anderen Mediums, sondern im Zuge einer allgemeinen Vertrauenskrise. Allerdings muss man zwischen den einzelnen Sendern unterscheiden: Im Wahlkampf 2005 in Deutschland sprachen jeweils über 40 Prozent der Befragten den öffentlich-rechtlichen Sendern ARD und ZDF glaubwürdige Berichterstattung zu, auf den nächstgereihten Privatsender RTL entfielen nur zwölf Prozent ${ }^{33}$.

In den USA hat die Glaubwürdigkeit des Fernsehens ebenfalls über die Jahre abgenommen. 1985 vertrauten noch rund 80 bis 85 Prozent den Nachrichten der großen Fernsehstationen und Tageszeitungen. Seit dem Jahr 2000 und zuletzt im Kongresswahljahr 2006 waren es höchstens 60 bis 65 Prozent bei den Fernsehanstalten und 55 Prozent bei den Tageszeitungen ${ }^{34}$. CNN hat von 42 Prozent Glaubwürdigkeit im Jahr 1998 auf 28 Prozent 2006 abgebaut $^{35}$. Dies könnte theoretisch als eine Entwicklung steigender Medienkompetenz bewertet werden (im Sinne einer kritischeren Grundhaltung Massenmedien gegenüber). Als wahrscheinlicher wird jedoch ein Zusammenhang mit generell sinkenden Vertrauenswerten in die Politik gesehen ${ }^{36}$.

29 Peter Filzmaier / Fritz Plasser, a.a.O. (Fn. 24), S. 104; vgl. auch The Pew Research Center for The People and The Press, Social networking and online videos take off. Internet's broader role in campaign 2008, Washington D.C. 2008, S. 4.

30 ORF-Medienforschung 2006 sowie eigene Berechnungen; vgl. auch Der Standard vom 26. März 2008, S. 29.

31 Allensbacher Computer- und Technikanalyse (ACTA) 2006.

32 Bertelsmann Stiftung (Hrsg.), Politische Partizipation in Deutschland. Ergebnisse einer repräsentativen Umfrage, Gütersloh 2004, S. 125.

33 http://www.presseportal.de/pm/7840/728701/zdf?search=forsa, Abruf am 26. März 2008.

34 Vgl. The Pew Research Center for The People and The Press, Media Consumption and Believability Study 2006, Washington D.C. 2006.

35 Ebenda, S. 46.

36 The Pew Research Center for The People and The Press, Public More Critical of Press, But Goodwill Persists, Washington D.C. 2005, S. 4. 


\title{
5. Fazit: Fernsehen als Leitmedium
}

Massenmedien sind, wie die Daten für Österreich und auch die internationalen Beispiele zeigen, als politische Informationsquelle in Wahlkampfzeiten nicht wichtiger oder glaubwürdiger als in Phasen ohne intensive Parteikampagnen. Unterschiede hinsichtlich soziodemographischer Daten gibt es in deutlichem Ausmaß nur bei der Nutzung des Internets, das sich als politische Informationsquelle der Jüngeren zeigt und als (neues) Medium an Bedeutung (noch) stetig gewinnt. Das Fernsehen ist demgegenüber das Medium einer älteren Generation, wobei es insgesamt das wichtigste Massenmedium für politische Informationen und Politikvermittlung darstellt. Zeitungen rangieren mit großem Abstand auf dem zweiten Platz. Die Politikberichterstattung im Radio hat einen radikalen Bedeutungsverlust erfahren.

Interessant ist zu beobachten, dass das Fernsehen (und in geringem Ausmaß auch das Radio) im Gegensatz zu den Zeitungen zuletzt an Glaubwürdigkeit verloren hat. Ob das mit einer kritischeren Grundhaltung des Publikums im Sinne von größerer Medienkompetenz zu tun hat oder vielmehr der Art der Vermittlung misstraut wird, muss an dieser Stelle dahingestellt bleiben (wenngleich Letzteres vermutet werden darf). Trotzdem bleibt das Fernsehen die mit Abstand wichtigste Informationsquelle für Politik. Auch international gesehen dominiert es in den westlichen Demokratien unabhängig von der Größe und Struktur des jeweiligen Medienmarktes. Jeder politische Akteur - egal von welcher Seite und mit welchem Ziel - muss das Interesse haben, in der Fernsehberichterstattung unterzukommen, und sich entsprechend auf die Spielregeln des Mediums einlassen. Das Fernsehen bestimmt damit nicht nur das politische Informationsverhalten auf Publikumsseite, sondern auch wesentlich die öffentliche Darstellung und Vermittlung von Politik durch die Akteure.

\section{Wahlsieg mit Ansage - und doch mit Überraschungen. Die italienischen Parlamentswahlen vom 13./14. April 2008}

\author{
Stefan Köppl
}

\section{Eine kurze Wahlperiode}

Den Parlamentswahlen von 2008 ging mit der 15. Legislaturperiode eine der kürzesten der italienischen Geschichte voraus, in der die 62. Nachkriegsregierung unter der Führung Romano Prodis die Geschicke des Landes lenkte, bis sie schließlich an internen Querelen zerbrach. Dabei trug die Regierungskoalition schon zum Zeitpunkt der Amtsübernahme den Keim des Scheiterns in sich. Im Vorfeld der letzten Parlamentswahlen hatte Prodi das bunte und fragmentierte Wahlbündnis Unione geschmiedet, um die Mitte-Rechts-Regierung Silvio Berlusconis abzulösen. So erreichte das Mitte-Links-Lager den Wahlsieg des Jahres 2006, der allerdings äußerst knapp ausfiel: Nur den mehrheitsbildenden Mechanismen des Wahlrechts 